\title{
Seasonal Variation in Klebsiella pneumoniae Blood Stream Infection: A Five Year Study
}

Fatima Khan, Naushaba Siddiqui ${ }^{\star}$, Asfia Sultan, Meher Rizvi, Indu Shukla and Haris M Khan

Department of Microbiology, Jawaharlal Nehru Medical College and Hospital, AMU, Aligarh, India

*Corresponding author: Naushaba Siddiqui, Department of Microbiology, Jawaharlal Nehru Medical College and Hospital, AMU, Aligarh, India, Tel: +919897520952; Email: naushsid@gmail.com

Received date: April 08, 2016; Accepted date: April 28, 2016; Published date: April 30, 2016

Copyright: (c) 2016 Khan F, et al. This is an open-access article distributed under the terms of the Creative Commons Attribution License, which permits unrestricted use, distribution, and reproduction in any medium, provided the original author and source are credited.

\begin{abstract}
Introduction: Klebsiella pneumoniae is a ubiquitous environmental organism and a common cause of serious gram-negative infections in humans. This study was conducted to examine the association between seasonal variation and the incidence rate of Klebsiella pneumoniae blood stream infection.

Material and methods: The retrospective study was conducted in the Department of Microbiology, JN Medical College AMU Aligarh for a period of 5 years from January 2011 to December 2015. Samples were received for blood culture in brain heart infusion broth. Cultures showing growth of Klebsiella pneumoniae were identified using standard biochemical procedures. Antimicrobial susceptibility testing was done on Mueller Hinton's agar by Kirby Bauer Disc diffusion method as per the CLSI guidelines. Detection of ESBL, AmpC and MBL production was done.

Results: $495(30.0 \%)$ isolates were found to be positive for Klebsiella pnuemoniae during the five year study period. Prevalence of Klebsiella pneumoniae isolates in blood stream infection for the warmest four months (JulySeptember) was found to be $61.8 \%$ as compared to $38.2 \%$ in the remaining year (January-June and OctoberDecember). Maximum number of Klebsiella pneumoniae were isolated the month of August $86(17.3 \%)$ followed by July $75(15.1 \%)$ while minimum isolates were found in the month of February $14(2.8 \%)$ and January $19(3.8 \%)$. ESBL producing isolates were $44(8.8 \%)$ and $318(64.20 \%)$ isolates were AmpC producers. $36(7.2 \%)$ isolates were found to be MBL producer.
\end{abstract}

Conclusion: Our study suggests that $K$. pneumoniae is an important pathogen in hospitals worldwide and that rates of $K$. pneumoniae blood stream infection vary seasonally.

Keywords: Klebsiella pneumonia; Infection; $A m p C$; Antimicrobial susceptibility; Cultures

\section{Introduction}

Seasonality is defined as a periodic surge in disease incidence corresponding to seasons or other predefined calendar periods [1]. As compared with community-acquired infections, for which seasonal variations have been identified, little is known about such variations. Infections caused by Gram-negative bacilli and healthcare associated infections. Seasonal variation in rates of infection with certain Gramnegative organisms including Acinetobacter species, Aeromonas hydrophila and Burkholderia pseudomallei has been previously described [2,3]. In addition, seasonal variation in bloodstream infection (BSI) due to Acinetobacter spp. and Klebsiella pneumoniae has been reported in studies from tertiary care centres [4].

Klebsiella pneumoniae is a ubiquitous environmental organism and a common cause of serious gram-negative infections in humans, including bloodstream infection, ventilator associated pneumonia, and urinary tract infection [5]. Infections due to $K$. pneumoniae occur in both endemic and outbreak settings [6]. Isolates of $K$. pneumoniae are becoming increasingly resistant to antibiotics and subsequently may become even more difficult to treat. We conducted this study to examine the association between seasonal variation and the incidence rate of Klebsiella pneumoniae BSI. The primary aim of the study was to compare the prevalence of Klebsiella pneumoniae BSI in the population between the warmest 4 months of the year (June through September) with that during the remainder of the months.

\section{Material and Methods}

The retrospective study was conducted in the Department of Microbiology, JN Medical College AMU Aligarh for a period of 5 years from January 2011 to December 2015. Samples were received for blood culture in brain heart infusion broth. Subcultures were done on $5 \%$ sheep Blood agar and Mac-Conkeys agar after 24 hours, 48 hours and 7 days of incubation at $37^{\circ} \mathrm{C}$. Cultures showing growth were identified by standard biochemical procedures [7]. Cultures showing growth of Klebsiella pneumoniae from blood (collected after 48 hours of hospital admission) with signs and symptoms of BSI (fever $>38^{\circ} \mathrm{C}$ ) were included in the study.

\section{Antimicrobial susceptibility testing}

Antimicrobial susceptibility testing was done on Mueller Hinton's agar by Kirby Bauer Disc diffusion method as per the Clinical and Laboratory Standards Institute guidelines (CLSI, 2014) for the following antimicrobials-amikacin $(30 \mu \mathrm{g})$, gentamycin $(10 \mu \mathrm{g})$, cefoperazone $(75 \mu \mathrm{g})$, ceftriaxone+sulbactum $(25 \mu \mathrm{g} / 75 \mu \mathrm{g})$, 
Citation: Khan F, Siddiqui N, Sultan A, Rizvi M, Shukla I, et al. (2016) Seasonal Variation in Klebsiella pneumoniae Blood Stream Infection: A

Page 2 of 4

cefoperazone+sulbactum $(75 \mu \mathrm{g} / 75 \mu \mathrm{g})$, levofloxacin $\quad(5 \mu \mathrm{g}), \quad$ Results piperacillin+tazobactum $(100 \mu \mathrm{g} / 10 \mu \mathrm{g})$ and imipinem $(10 \mu \mathrm{g})$.

\section{Detection of extended spectrum beta lactamases}

Screening of possible ESBL production was done by using ceftriaxone $(30 \mu \mathrm{g})$ and cefoperazone $(75 \mu \mathrm{g})$. Those isolates with zone diameters less than $25 \mathrm{~mm}$ for ceftriaxone and less than $22 \mathrm{~mm}$ for cefoperazone were subsequently confirmed for ESBL production.Confirmation was done by noting the potentiation of the activity of cefoperazone in the presence of cefoperazone sulbactum [8].

\section{Detection of inducible and derepressed $A m p C$ beta lactamase}

Detection of $A m p C$ betalactamase was done on isolates resistant to ceftriaxone $(30 \mu \mathrm{g})$, cefixime $(15 \mu \mathrm{g})$, cefoperazone $(75 \mu \mathrm{g})$ and cefoperazone sulbactum $(75 / 75 \mu \mathrm{g})$. Induction of $A m p C$ synthesis was based on the disc approximation assay using imipenem as inducer [8].
A total of 18,604 samples were received for blood culture during the five year period. 1648 (9.0\%) samples were found positive on culture. Out of these samples, 495 (30.0\%) isolates were found to be positive for Klebsiella pnuemoniae during the five year study period. The other pathogens isolated during this period were Staphylococcus aureus (295, 17.9\%), coagulase negative Staphylococcus species (200, 12.1\%), Enterococcus faecalis (35, 2.1\%), Pseudomonas species (157, 9.5\%), Acinetobacter species (120, 7.2\%), Citrobacter species (156, 9.4\%), Escherichia coli (123, 7.4\%), and Salmonella typhi (67, 4.1\%) (Figure 1). Out of these 495 isolates positive for Klebsiella pnuemoniae, 212 $(42.8 \%)$ strains were isolated in the year 2015, 107 (21.6\%) in 2014, 80 (16.1\%) in 2013, $53(10.7 \%)$ in 2012 and $43(8.6 \%)$ isolates in 2011 (Figure 2). There was a steep rise in the Klebsiella pneumoniae isolates from $8.68 \%$ in the year 2011 to $42.8 \%$ in the year 2015. Majority of the isolates were from paediatric age group.

\section{Detection of metallobeta-lactamases}

Detection of MBL was done by Hodge test and Double Disc synergy test using EDTA. The method was as described by Lee et al. [9].

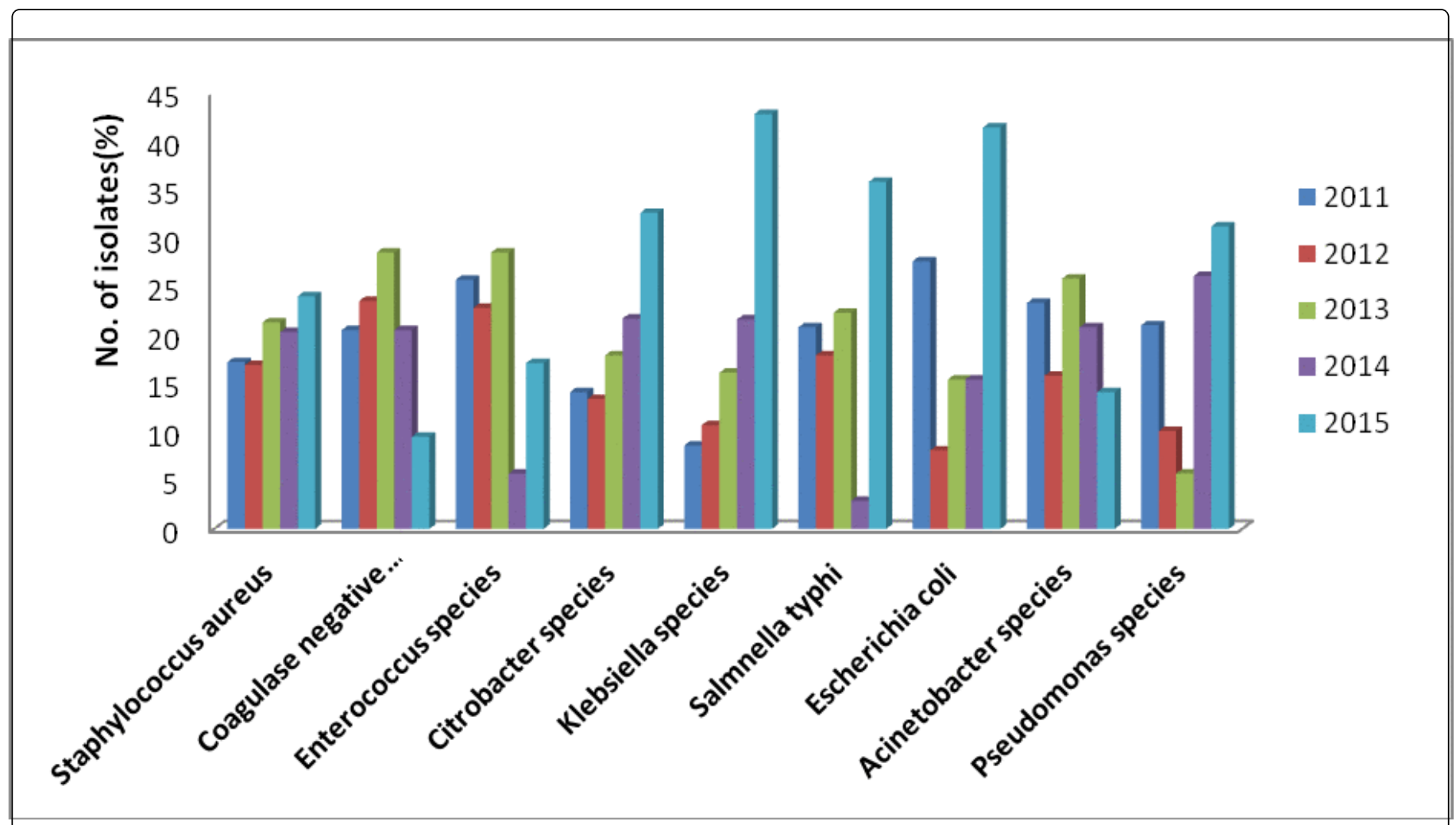

Figure 1: Distribution of bacteria isolated from blood culture for the period of five years. 
Citation: Khan F, Siddiqui N, Sultan A, Rizvi M, Shukla I, et al. (2016) Seasonal Variation in Klebsiella pneumoniae Blood Stream Infection: A

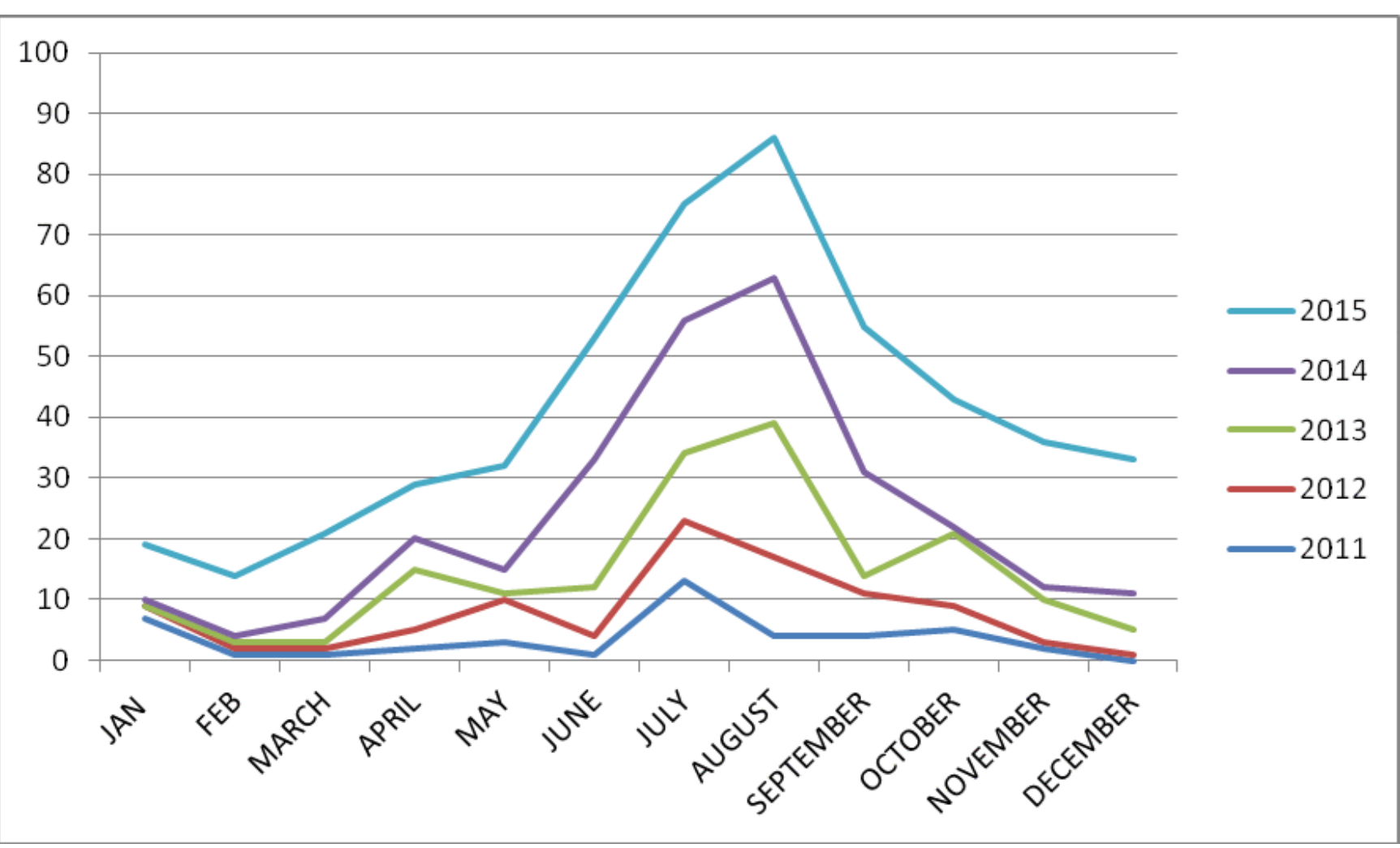

Figure 2: Monthwise distribution of Klebsiella pneumoniae in blood culture for the period of 5 years.

Prevalence of Klebsiella pneumoniae isolates in blood stream infection for the warmest four months (July-September) was found to be $61.8 \%$ as compared to $38.2 \%$ in the remaining year (January-June and October-December). Maximum number of Klebsiella pneumoniae were isolated the month of August 86 (17.3\%) followed by July 75 (15.1\%) while minimum isolates were found in the month of February $14(2.8 \%)$ and January $19(3.8 \%)$ (Table 1$)$.

\begin{tabular}{|c|c|c|c|c|c|c|}
\hline Time Period & \multicolumn{6}{|c|}{ No. of Isolates } \\
\hline & $2011(\mathrm{n}=43)$ & $2012(\mathrm{n}=53)$ & $2013(\mathrm{n}=80)$ & $2014(\mathrm{n}=107)$ & $2015(\mathrm{n}=212)$ & Total $(\mathrm{N}=495)$ \\
\hline June-September & $22(51.1 \%)$ & $33(62.2 \%)$ & $42(52.5 \%)$ & $83(77.5 \%)$ & $126(59.4 \%)$ & $306(61.8 \%)$ \\
\hline Rest of the Year & $21(48.9 \%)$ & $20(37.8 \%)$ & $38(47.5 \%)$ & $24(22.5 \%)$ & $86(40.6 \%)$ & $189(38.2 \%)$ \\
\hline
\end{tabular}

Table 1: Prevalence of Klebsiella pneumoniae isolates in blood stream infection for the warmest 4 months as compared to remainder of the months.

On antimicrobial sensitivity testing maximum resistance $(88.10 \%)$ was shown to the $\beta$-lactam group of antimicrobials. Aminoglycosides also had poor activity with resistance to $77.38 \%$ of the isolates. Flouroquinolones had better activity with resistance to only $39.39 \%$ of the isolates. Sensitivity to Levofloxacin remained uniform through all the five years while that of cephaolosporins showed drastic fall through the years ( $23 \%$ in 2011 to $2.35 \%$ in 2105 ). There was no change in resistance pattern in the warmest four months and remainder of the year. $21(4.24 \%)$ isolates were found to be multidrug resistant. ESBL producing isolates were $44(8.8 \%)$ and $318(64.20 \%)$ isolates were $A m p C$ producers. 36 (7.2\%) isolates were found to be MBL producer. There was an immense increase in resistance over the years. More than fifty percent of the $A m p C$ producers (162 out of 318) and MBL producers ( 25 out of 36 ) were isolated in the year 2015.

\section{Discussion}

Klebsiella pneumoniae causes serious, life-threatening infections in humans in endemic and epidemic settings. Our study is probably the first study in North India to describe a seasonal variation in the prevalence of $K$. pneumoniae blood stream infection in humans. In our study, the rate of $K$. pneumoniae BSI was 1.6 times higher during the 4 warmest months of the year as compared to the rest of the year. Our data suggest that rates of $K$. pneumoniae blood stream infection were associated with changes in temperature and humidity. The exact cause 
Page 4 of 4

for the observed higher rates of $K$. pneumonia BSI during warm months remains elusive.

Several previously described characteristics of $K$. pneumoniae support our findings [4]. First, $K$. pneumoniae is the most heat tolerant of all enteric pathogens [10]. Second, the specific growth rate of $K$. pneumoniae is maximal at temperatures approaching $36.9^{\circ} \mathrm{C}$ [11]. Finally, $K$. pneumoniae survives better at higher humidity, as experimental models have shown that dehydration is an important factor in inactivating the organism [12].

$K$. pneumoniae is found naturally in 2 habitats: in environmental settings, such as water, sewage, soil, and plants, and on the mucosal surfaces of mammals. The density of $K$. pneumoniae in the environment (e.g., in freshwater ponds) is higher during warm months. Furthermore, $K$. pneumoniae mammary infections in cows are more common during summer [13]. Thus, it is reasonable to hypothesize that humans also have higher levels of colonization with environmental $K$. pneumoniae during warm months. These environmental $K$. pneumoniae isolates are just as virulent as clinical isolates from hospitals and can produce important virulence factors $[14,15]$. Furthermore, intestinal colonization typically precedes infection in humans, and hospitalized patients with $K$. pneumoniae colonization are at 4 -fold higher risk of $K$. pneumoniae infection than non-carriers. Thus, increased colonization leading to infection may explain the observed increase in infection rate in summer [15].

Our study highlights an increase in the isolation of multidrug resistant $K$. pneumonia in blood. In our study, 21 (4.24\%) isolates were found to be multidrug resistant with $8.8 \%$ of isolates being ESBL producers, $64.20 \% A m p C$ producers and $7.2 \%$ MBL producer. Similar results were observed in other studies $[6,16,17]$. Our study showed a drastic rise in resistance over the years. Fifty one percent of the $A m p C$ producers and sixty nine percent of the MBL producers were isolated in the year 2015. However there was no change in resistance pattern with seasonal variation. The increase in antibiotic resistance is due to several factors but the major cause appears to be excessive use of antibiotics [18]. Injudicious use of $\beta$-lactam group of antimicrobials, commonly used for treating Klebsiella pneumonia infection over the years had led to development of resistant mutants to these drugs. However certain drugs like levofloxacin which were less commonly used maintain better sensitivity profile. One possible solution has been to call an "antibiotic holiday" for commonly used drugs in which a drug is retired for a while until the mutant strain of bacteria dies off and the antibiotic can be used again.

In summary, our study suggests that $K$. pneumoniae is an important pathogen in hospitals worldwide and that rates of $K$. pneumoniae blood stream infection vary seasonally. Our novel study should further stimulate hypothesis-generated investigations to evaluate further the impact of climate on infectious diseases. Furthermore, our study highlights that a sudden increase in the isolation of multidrug resistant pathogens should be taken as a challenge and prompt action should be taken. There is an urgent need for early detection of these isolates for better treatment outcomes. Rational and judicious use of all antimicrobial agents and infection control policies are of paramount importance in curtailing this growing epidemic.

\section{References}

1. Richet H (2012) Seasonality in Gram-negative and healthcare-associated infections. Clin Microbiol Infect 18: 934-940.

2. McDonald LC, Banerjee SN, Jarvis WR (1999) Seasonal variation of Acinetobacter infections: 1987-1996. Nosocomial Infections Surveillance System. Clin Infect Dis 29: 1133-1137.

3. Currie BJ, Fisher DA, Howard DM, Burrow JN, Selvanayagam S, et al (2000) The epidemiology of melioidosis in Australia and Papua New Guinea. Acta Trop 74: 121-127.

4. Anderson DJ, Richet H, Chen LF, Spelman DW, Hung YJ, et al. (2008) Seasonal variation in Klebsiella pneumoniae bloodstream infection on 4 continents. J Infect Dis 197: 752-756.

5. Skogberg K, Lyytikäinen O, Ruutu P, Ollgren J, Nuorti JP (2008) Increase in bloodstream infections in Finland, 1995-2002. Epidemiol Infect 136: 108-114.

6. Khan F, Siddiqui N, Sultan A, Rizvi M, Abqari S, et al. (2015) Klebsiella pneumoniae Outbreak in Paediatric Ward: Detection and Prevention. Int J Curr Microbiol App Sci 1: 81-87.

7. Collee JG, Fraser AG, Marmion BP, Simmons MA (2006) McCartney practical medical microbiology. In: Collee JG, Miles RS, Watt B (eds.) Tests for the identifi cation of Bacteria, (14th edn.) Elsevier, New Delhi, India.

8. Rizvi M, Fatima N, Rashid M, Shukla I, Malik A, et al. (2009) Extended spectrum AmpC and metallo-beta-lactamases in Serratia and Citrobacter spp. in a disc approximation assay. J Infect Dev Ctries 3: 285-294.

9. Lee K, Chong Y, Shin HB, Kim YA, Yong D, et al. (2001) Modified Hodge test and EDTA disc synergy tests to screen metallo beta lactamase producing strains of Pseudomonas and Acinetobacter species. Clin Microbiol Infect 7: 88-91.

10. Esener AA, Roels JA, Kossen NWF (1981) The influence of temperature on the maximum specific growth rate of Klebsiella pneumoniae. Biotechnol Bioengineering 23: 1401-1405.

11. Esener AA, Roels JA, Kossen NW (1983) The influence of temperature on the energetics of Klebsiella pneumoniae. Biotechnol Bioeng 25: 2093-2098.

12. Al-Harbi AH (2003) Faecal coliforms in pond water, sediments and hybridtilapia Oreochromis niloticus_Oreochromis aureus in Saudi Arabia. Aquaculture Res 34: 517-524.

13. Podschun R, Ullmann U (1998) Klebsiella spp. as nosocomial pathogens: epidemiology, taxonomy, typing methods, and pathogenicity factors. Clin Microbiol Rev 11: 589-603.

14. Struve C, Krogfelt KA (2004) Pathogenic potential of environmental Klebsiella pneumoniae isolates. Environ Microbiol 6: 584-590.

15. Podschun R, Pietsch S, Höller C, Ullmann U (2001) Incidence of Klebsiella species in surface waters and their expression of virulence factors. Appl Environ Microbiol 67: 3325-3327.

16. Rastogi V, Nirwan PS, Jain S, Kapil A (2010) Nosocomial outbreak of septicaemia in neonatal intensive care unit due to extended spectrum Blactamase producing Klebsiella pneumoniae showing multiple mechanisms of drug resistance. Indian J Med Microbiol 28: 380-384.

17. Viswanathan R, Singh AK, Mukherjee S, Mukherjee R, DAS P, et al. (2011) An outbreak of neonatal sepsis presenting with exanthematous rash caused by Klebsiella pneumoniae. Epidemiol Infect 139: 226-228.

18. Kotwani A, Holloway K, Chaudhury RR (2009) Methodology for surveillance of antimicrobials use among out-patients in Delhi. Indian J Med Res 129: 555-560. 